\title{
TITLE: ELECTRON BEAM SENSITIVITY STUDY OF THE LOS ALAMOS ADVANCED FREE-ELECTRON LASER BEAMLINE
}

AUTHOR(S): S. Hartman, A. Lombardi, R. Sheffield, and T. Wang

\author{
SUBMITTED TO: Thirteenth International Free-Electron Laser Conference \\ Santa Fe, NM \\ August 25-30, 1991
}

\section{DISCLAIMER}

\begin{abstract}
This report was prepared as an account of work sponsored by an agency of the United States Government. Neither the United States Government nor any agency thereof, nor any of their employees, makes any warranty, express or implied, or assumes any legal liability or responsibility for the accuracy, completeness, or usefulness of any information, apparatus, product, or process disclosed, or represents that its use would not infringe privately owned rights. Reference herein to any specific commercial product, process, or service by trade name, trademark, ence herein to any specise does not necessarily constitute or imply its endorsement, recommendation, or favoring by the United States Government or any agency thereof. The views and opinions of authors expressed herein do not necessarily state or reflect those of the United States Government or any agency thereof.
\end{abstract}

By acceptance of this article, the publisher recognizes that the U.S. Govemment retains a nonexclusive, royalty-free license to publish or reproduce the published form of this contribution, or to allow others to do so, for U.S. Government purposes.

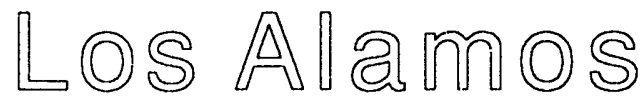

Los Alamos National Laboratiory

Los Alamos, New Mexico 87'545 


\title{
ELECTRON-BEAM SENSITIVITY STUDY
}

\author{
OF THE LOS ALAMOS ADVANCED FREE-ELECTRON LASER BEAM LINE * \\ Spencer Hartman, ${ }^{* *}$ Alessandra Lombardi, \\ Richard Sheffield and Tai-Sen Wang \\ Los Alamos National Laboratory \\ Mail Stop H829 \\ Los Alamos, NM 87545 \\ ** University of California at Los Angeles \\ Dept. of Physics, 405 Hilgard Ave., Los Angeles, CA 90024
}

\begin{abstract}
A sensitivity study that used the particle tracking code PARMELA was performed on the Advanced Free-Electron Laser (AFEL) [1]. The tolerances on the alignment of the beam-line elements and the magnetic-field strengths were examined.

Two base-line linac configurations were determined at the beginning of the study. The electron beam was then matched into the wiggler. The optimized beam-line parameters were varied independently and their sensitivities were judged with the criterion that the free-electron laser (FEL) effective-detuning parameter should not fluctuate more than $10 \%$.

The results of this study set the specifications for the alignment, for the sensitivity of the steering magnets, and for the accuracy of the magnetic field in the quadrupoles and dipoles.
\end{abstract}

* Work supported by Los Alamos National Laboratory Institutional Supporting Research, under the auspices of the United States Department of Energy.

Proof to be sent to:Alessandra Lombardi, MS H829, Los Alamos National Laboratory, Los Alamos, NM 87545, U.S.A. Phone (505)667-9426, fax (505)667-0919 
ELECTRON-BEAM SENSITIVITY STUDY

\title{
OF THE LOS ALAMOS ADVANCED FREE-ELECTRON LASER BEAM LINE *
}

\author{
Spencer Hartman,** Alessandra Lombardi, \\ Richard Sheffield and Tai-Sen Wang \\ Los Alamos National Laboratory \\ Mail Stop H825 \\ Los Alamos, NM 87545 \\ ** University of California at Los Angeles \\ Dept. of Physics, 405 Hilgard Ave., Los Angeles, CA 90024
}

\section{INTRODUCTION}

The Advanced Free-Electron Laser (AFEL) [1] is a project at the Los Alamos National Laboratory, that aims to build a compact, high-performance free-electron laser. The high brightness beam required, a less-than-10 $\pi$-mm-mrad-emittance , 300-A-peak-current electron beam, will be produced by a photocatode and accelerated to $20 \mathrm{MeV}$ by a $1.2-\mathrm{m}$-long, linear accelerator. An optical beam-line, consisting of seven quadrupoles and two dipoles, will guide the beam from the accelerator to the wiggler. Figure 1 shows the complete beam-line of the AFEL experiment.

In this paper we will present a sensitivity study of the components of the AFEL beam-line. The results of this study set the specifications for the alignment, for the sensitivity of the steering magnets, and for the accuracy of the magnetic field in the quadrupoles and dipoles.

\section{EFFECTIVE DETUNTNG PARAMETER}

The sensitivity of a beam-line specific parameter is defined by the impact of the change of this parameter on the gain in the AFEL interaction. 
An indirect measure of the quality of the FEL interaction is the effective detuning parameter [2] which is defined as follows :

$$
\Delta \nu=\frac{2 \pi h N}{\left(1+K_{w}^{2}\right)}\left(\left(K_{w} K r\right)^{2}+(\gamma \theta)^{2}\right)+\frac{4 \pi h N\left(\gamma-\gamma_{0}\right)}{\gamma_{\circ}}
$$

where

$h$ is the harmonic number

$\mathrm{N}$ is the number of wiggler periods

$K_{w}$ is the wiggler wave-number

$\mathrm{K}$ is the wiggler parameter

$r$ is the rms electron-beam radius

$\gamma$ is the particle energy in rest-mass energy units

$\gamma_{\circ}$ is the average bunch energy

$\theta$ is the injection angle into the wiggler.

The two terms in Eq. (1) represent, respectively, the matching into the wiggler and the energy spread of the beam, which are two determinant quantities for the FEL performance. The quantity $\Delta \nu$ is related to the spontaneous emission intensity spectrum by the relation $\left(\frac{\sin (\Delta \nu)}{\Delta \nu}\right)^{2}$.

We should take into account that the $\nu$ parameter is a good representation of the FEL interaction only when the electron beam and optical beam overlap completely. Consider figure $2 a, b$ and $c:$ in the first two cases $\nu$ is a good representation of the FEL process. In the third case the FEL has a loss in gain because of the mismatch that is not considered in the $\nu$ parameter.

To calculate $\Delta \nu$ we first use the particle tracking code PARMELA [3] to simulate the electron dynamics from the photocathode through the accelerator and the transport line, 
and we extract the particles canonical coordinates at selected points in the wiggler. Shen, with a special postprocessor, we slice the beam into equal-sized bins of the slippage length and calculate average energy $(\gamma)$ and radius $(r)$ for each bin. When we put these values into Eq. (1), we obtain a $\Delta \nu$ value for each bin; the final $\Delta \nu$ is the average over all the bins.

Beam-line Auctuations tolerated are those that do not change the average $\Delta \nu$ by more than $10 \%$ with respect to the base-line case.

\section{PROCEDURE FOR THE NUMERICAL SIMULATION}

The simulations were done in three steps : first PARMELA was run to track the beam from the photocathode to the end of the linac. Then the linac parameters were optimized. Finally a sensitivity study was carried out for the accelerating line [4].

Two initial linac configurations were chosen, and the sensitivity of the optics transport line was analyzed.

A schematic layout of the beam-line optics elements is reported in Figure 2. The magnetic-elements field strengths are reported in Table 1. The physics underlying the design of this beam-line can be found in [5].

The magnetic field strengths of the quadrupoles and dipoles were varied independently.

The transverse displacements were done with the quadrupole doublets being moved as a single structure while the two dipoles and the fifth quadrupole $\left(Q_{5}\right)$ were varied independentely. The doublets were treated as a single unit because they will be permanentely mounted together on the same support structure and once placed they should not move relative to each other. However, the effects of rotating a single quadrupole was also calculated. Only a single parameter was varied for each sensitivity run. The calculations were carried on the third harmonic of the wiggler $(\lambda=1.2 \mathrm{~mm})$; the tolerance for the first harmonic is looser as the beam emittance requirements are less strict.

The beam properties and wiggier parameters are given in Table 2. 
The simulations did not incorporate the effect of thermal emittance and wiggler focusing. These two factors should not appreciabily affect the beam-line sensitivity.

Also, the simulations did not take into account the possible loss in gain due to a not perfect ovelapping between the optical and the electron beam. To give an estimate of this effect, we calculated the position of the centroid and the radius of the beam along the wiggler, and we compared them to the position and dimension of a gaussian optical beam. We found that in the worst case we suffered a loss in the overlapping area, on the third harmonic, of less than $1 / 10$. We think that we can correct this problem with steering magnets (see Figure 1). However this problem is more dramatic on higher harmonics.

\section{RESULTS}

Table 3 summarizes the results of approximately 150 PARMELA runs and should give a reasonable idea of the critical parameters that need special attention in the set up of the electron-beam transport optics, i.e., micropositioning of elements, setting field strengths, minimizing element skew, and so forth.

The $\mathrm{x}$-direction or direction of dispersion is much more sensitive than the $\mathrm{y}$ direction. These results indicate that we must align the beam-line elements to within at least 100 $\mu \mathrm{m}$ or better. Also, taking into account the centroid displacement we must steer the beam with an accuracy of the order of $50 \mu \mathrm{m}$ to ensure a complete overlap between the optical and electron beam.

We also tried another linac configuration where the solenoid strength was changed by $10 \%$ with respect to the previous case. In this beam-line configuration the AFEL was extremely sensitive. This was evidenced by loosing the beam when we changed the B-field strength in the first quadrupole by only $5 \%$. This abrupt loss of the beam lead us to the conclusion that this beam-line is too sensitive and further study is not worthwhile. 


\section{CONCLUSIONS}

To limit the fluctuation in the AFEL $\Delta \nu$ parameter to less than $10 \%$ on the third harmonic, the transverse alignment of the beam-line elements needs to be done to within $100 \mu \mathrm{m}$, with the added requirement that the bearn can be steered and monitored to within $50 \mu \mathrm{m}$. The magnetic-field strengths should be stable to within $0.1 \%$ and the rotation tolerance about the $z$-axis of the magnetic elements is $0.1^{\circ}$. However the two quadrupoles that constitute the first doublet should be aligned to within $0.01^{\circ}$. 


\section{REFERENCES}

[1] K.C.D. Chan et al., "Los Alamos advanced Free-Electron Laser", these proceedings.

[2] W.B. Colson, G. Dattoli, and F. Ciocci, "Angular gain spectrum of free-electron lasers", Phys. Rev. A 64,2 (1985).

[3] PARMELA is a computer code written by $K$. Crandell and is available from the Los Alamos Accelerator Code Group, AT-7, ms H829, Los Alamos National Laboratory, Los Alamos, NM 87545, U.S.A.

[4] R.L. Sheffield, "Sensitivity study of the AFEL accelerator", Los Alamos National Laboratory technical memorandum AT-7:91-TM-5.

[5] T.F. Wang et al., " Design of the e-beam transport for the AFEL", these proceedings. 


\section{FIGURE CAPTIONS}

Fig. 1. Schematic of the AFEL experiment beam-line.

Fig. 2. Relative positions of the optical and electrical beam in the wiggler.

Fig. 3. Schematic of the AFEL beam-line used in the simulations. 


\section{Table 1}

Magnetic-field value in the base-line case.

$$
\begin{aligned}
& Q_{1} 4730 \mathrm{G} / \mathrm{cm} \\
& Q_{2}-3690 \mathrm{G} / \mathrm{cm} \\
& Q_{3} 4327 \mathrm{G} / \mathrm{cm} \\
& Q_{4}-2680 \mathrm{G} / \mathrm{cm} \\
& D_{1} 3521 \mathrm{G} \\
& Q_{3} 1517 \mathrm{G} / \mathrm{cm} \\
& D_{2} 3521 \mathrm{G} \\
& Q_{6}-3011 \mathrm{G} / \mathrm{cm} \\
& Q_{7} 3339 \mathrm{G} / \mathrm{cm}
\end{aligned}
$$


Table 2

FEL parameters.

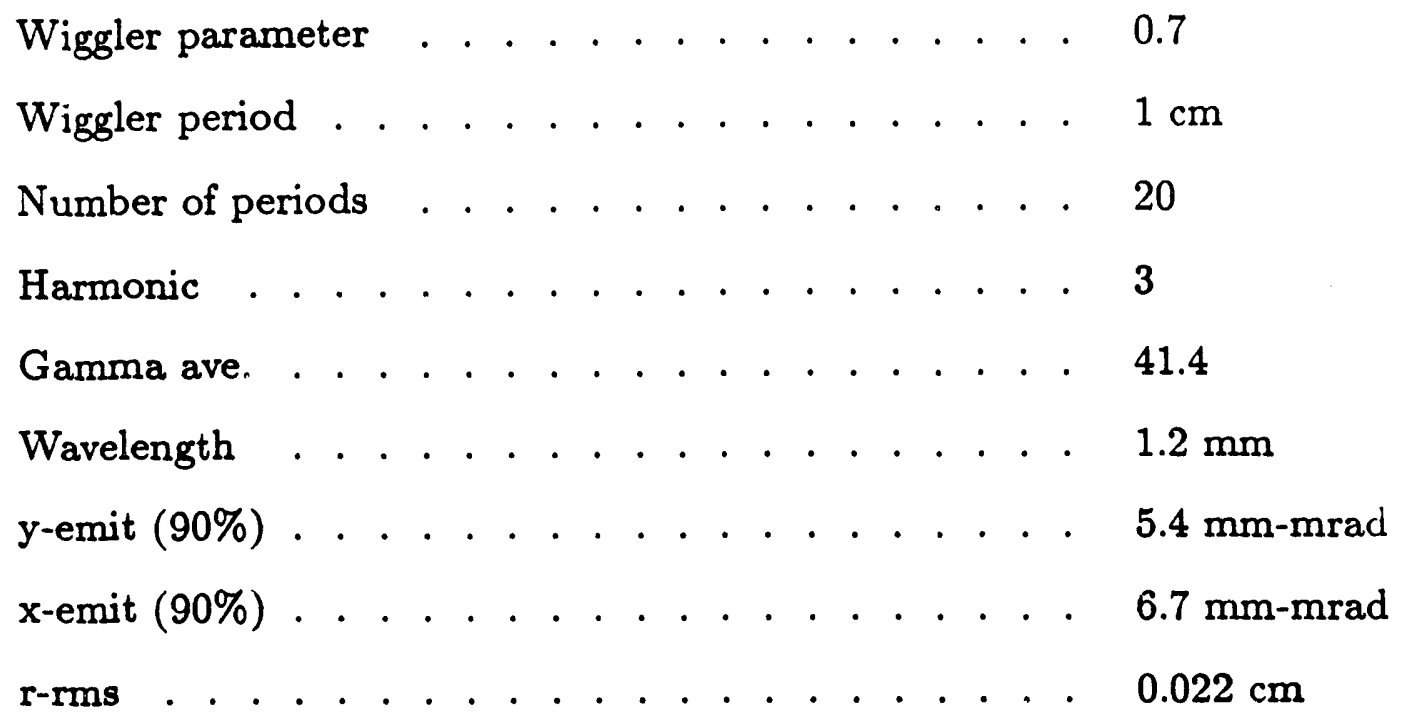


Table 3

Beam-line tolerances

$\begin{array}{llll}\text { B-field } & \mathrm{x} \text {-mis } & \mathrm{y} \text {-mis } & \text { rot } \\ ( \pm \%) & (\mu \mathrm{m}) & (\mu \mathrm{m}) & (\mathrm{deg})\end{array}$

$\begin{array}{lllll}\text { Q1 } & 2 & 100 & 300 & 0.01 \\ \text { Q2 } & 0.5 & & & 0.01 \\ & & & & \\ \text { Q3 } & 0.1 & 100 & 600 & 0.1 \\ \text { Q4 } & 0.5 & & & 0.1\end{array}$

$\begin{array}{lllll}\text { D1 } & 10 & 400 & 400 & 0.1\end{array}$

$\begin{array}{lllll}\text { Q5 } & 0.1 & 100 & 400 & 0.1\end{array}$

$\begin{array}{lllll}\text { D2 } & 5 & 400 & 400 & 0.1\end{array}$

$\begin{array}{lllll}\text { Q6 } & 2 & 400 & 300 & 0.1 \\ \text { Q7 } & 2 & & & 0.1\end{array}$

$\begin{array}{llll}\text { Linac } & 600 & 600 & 0.1\end{array}$ 


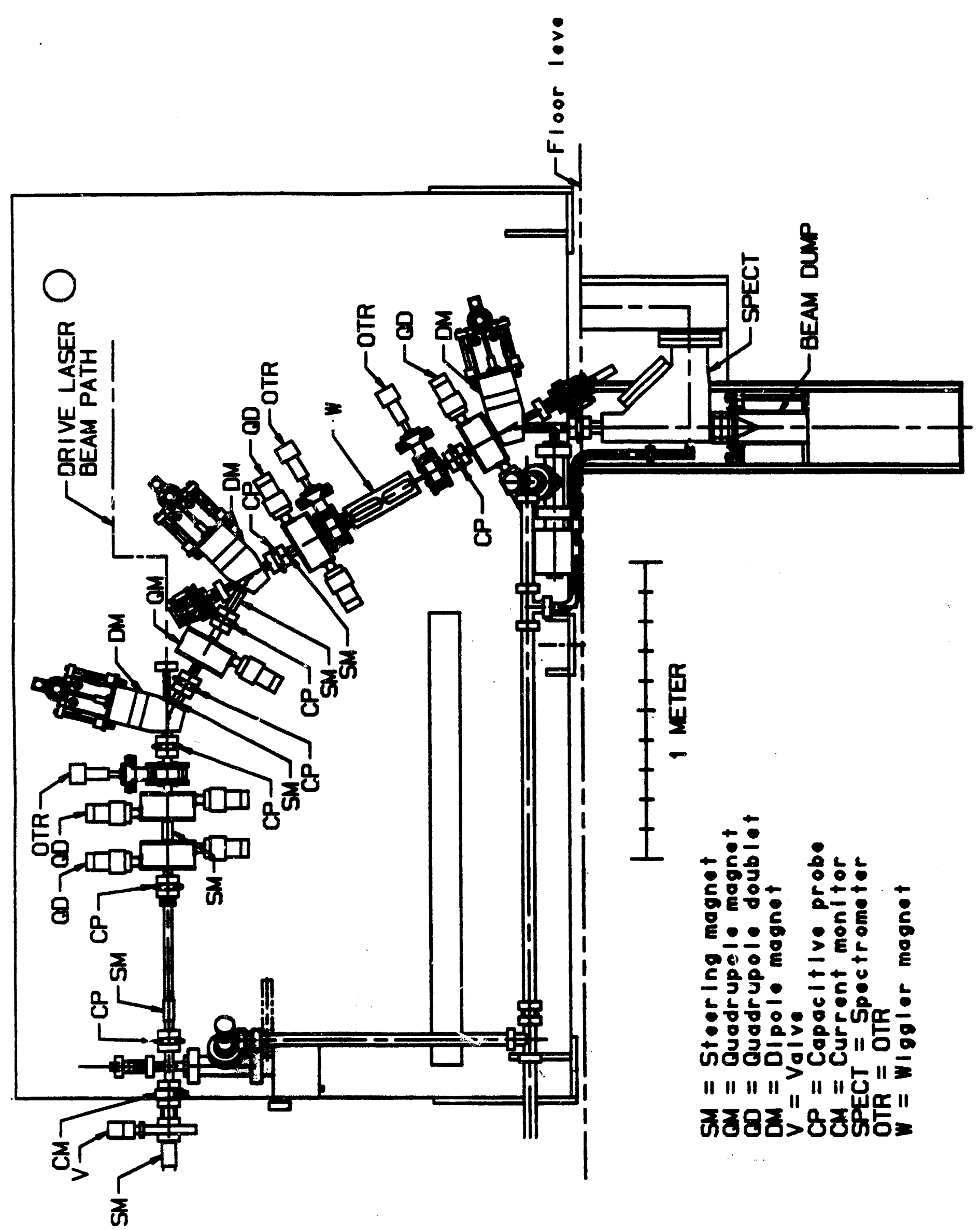




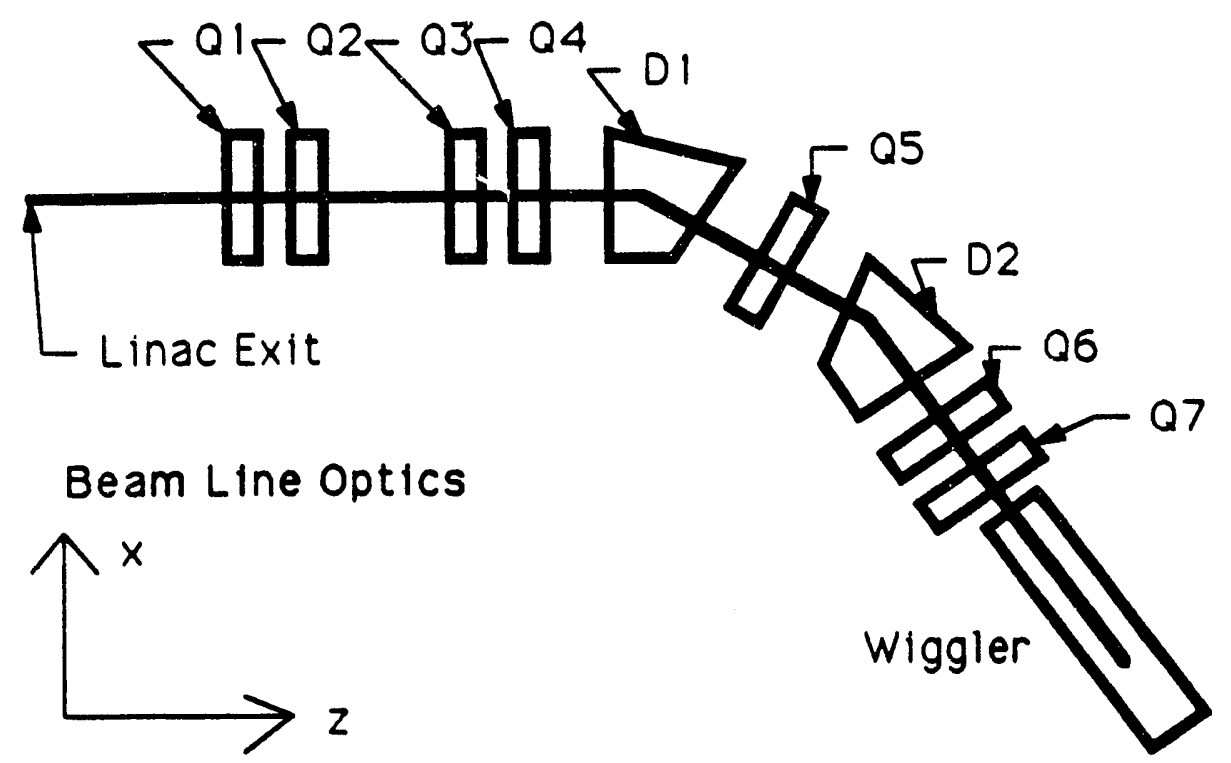



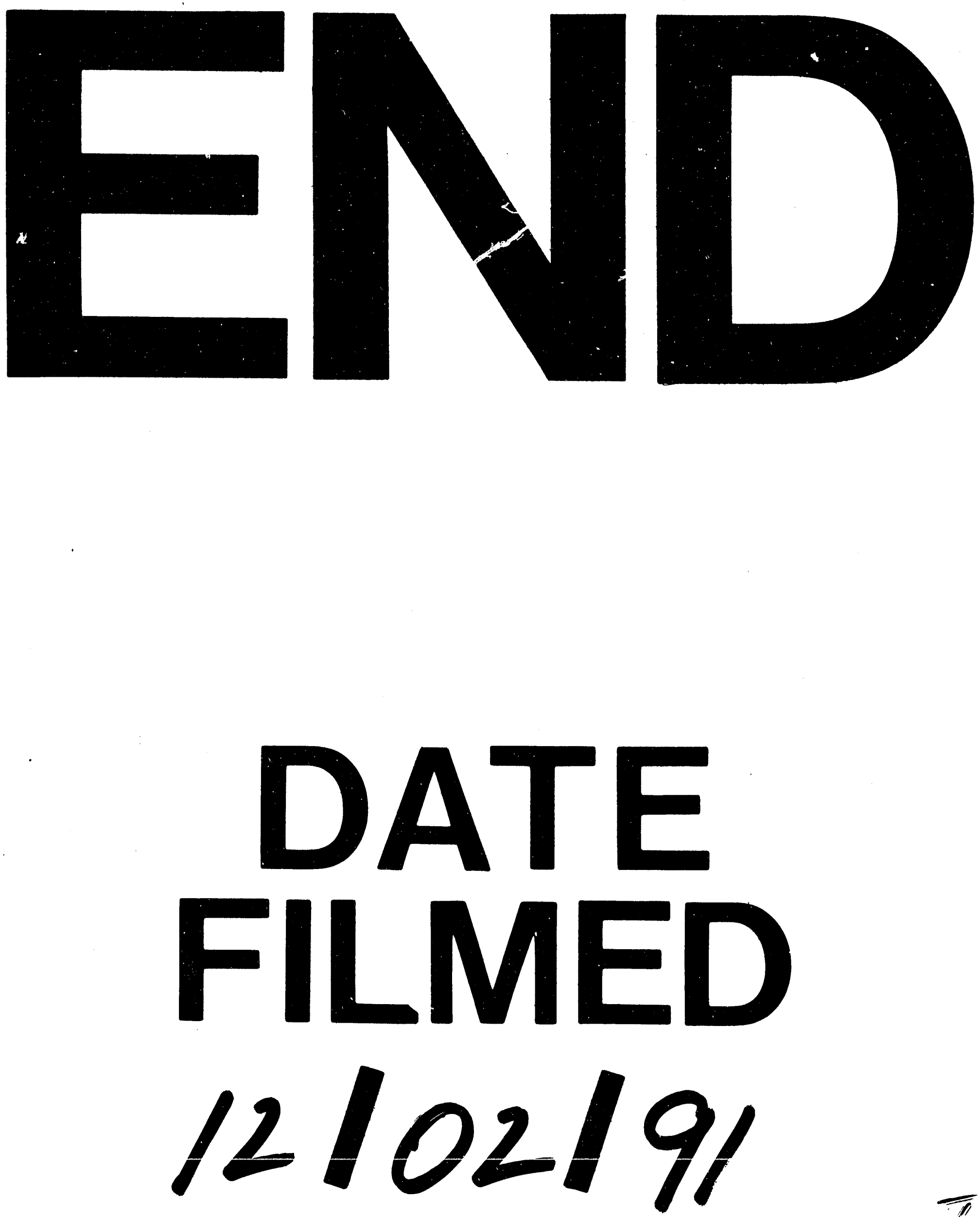
